\title{
Tratamiento fisioterapéutico en un niño de cinco años con angiomatosis cutáneo meningoespinal asintomática
} Physiotherapeutic treatment in a five-year-old boy with asymptomatic meningospinal cutaneous angiomatosis

Luzmila Olivera-Huerta ${ }^{1}$

\section{Resumen}

El Síndrome de Cobb (cutáneo angiomatosis) es una patología poco frecuente de etiología congénita no hereditaria, se caracteriza por presentar anomalías vasculares en la médula espinal, involucrando también a la piel, músculos y huesos. Este caso reporta a un niño de cinco años diagnosticado con Síndrome de Cobb que después de la intervención quirúrgica a causa de una caída manifestó síntomas de dolor intenso en la zona lumbar. El objetivo de la intervención fisioterapéutica fue mejorar el proceso de transición de sedente a bípedo. Para ello, se emplearon las técnicas del enfoque Perfetti, concepto Bobath, estiramiento estático pasivo, pasivo asistido, estimulación exteroceptiva y ejercicios propioceptivos e isométricos. Entre los resultados se destacan principalmente la mejora del proceso de trans ición de sedente a bipedo, la recuperación de la elasticidad muscular y la flexibilidad articular. Respecto a la sensibilidad, se aumentó la percepción superficial y propioceptiva en general. Asimismo, se ganó mayor fuerza muscular y se recuperó el trofismo muscular. Con la intervención fisioterapéutica realizada se logró mayor independencia respecto a la movilización y el desarrollo de las actividades de la vida diaria.

Palabras clave: Neuropatías; Angiomatosis; Malformaciones arteriovenosas; Neurocirugía; Embolización (Fuente: DeCS).

\begin{abstract}
Cobb syndrome (cutaneous angiomatosis) is a rare condition of non-hereditary congenital etiology. It is characterized by vascular anomalies in the spinal cord, also involving the skin, muscles, and bones. This case reports a five-year-old boy diagnosed with Cobb Syndrome who, after the surgical intervention due to a fall, manifested symptoms of intense pain in the lower back. The objective of the physiotherapeutic intervention was to improve the process of transition from seated to biped. For this, the techniques of Perfetti approach, Bobath concept, passive static stretching, assisted passive, exteroceptive stimulation and proprioceptive and isometric exercises were used. Among the results, we can highlight the improvement of the process of transition from seated to bipedal, the recovery of mu scular elasticity and joint flexibility. Regarding sensitivity, the superficial and proprioceptive perception was increased in general. Also, greater muscle strength was gained and muscle tropism recovered. With the physiotherapeutic intervention carried out, greater independence was obtained with respect to the mobilization and development of the activities of daily life.
\end{abstract}

Keys words: Neuropathies; Angiomatosis; Arteriovenous malformations; Neurosurgery; Embolization (Source: DeCS).

Para citar:

Olivera L. Tratamiento fisioterapéutico en un niño de cinco años con angiomatosis cutáneo meningoespinal asintomática . CASUS. 2019;4(1):45-53.

${ }^{1}$ Clínica San Juan de Dios. Bachiller de Terapia Física. Correo electrónico: moliverauni2013@gmail.com
Fecha de recepción: 11-02-19

Fecha de envío a pares: 15-02-19

Fecha de aprobación por pares : 07-04-19

Fecha de aceptación: 09-04-19 


\section{INTRODUCCIÓN}

El síndrome de Cobb denominado también como angiomatosis cutáneovertebralmedular pertenece al grupo de síndromes neurocutáneos asociada a angiomas espinales, malformaciones vasculares (MV) y lesiones cutáneas en el mismo dermatoma $(1,2)$. Su ocurrencia es poco frecuente y de etiología congénita no hereditaria $(1,2)$. Las MV se subdividen en: malformaciones de flujo bajo o lento y de flujo rápido o alto. Los de alto flujo incluyen componentes arteriales, dolor, ulceración, cambios isquémicos, sangrado, insuficiencia cardíaca congestiva. Los de flujo bajo presentan combinaciones de capilares venosos y linfáticos, pueden detectarse al nacer o posteriormente (3).

Este síndrome es bastante variable y se caracteriza por estar presente en la médula espinal, puede afectar desde la C2-L (4), con una tríada de piel, músculo y hueso. Además de la afectación de la vasculatura asociada al cuerpo, a veces va acompañada de angiomatosis visceral $(2,5,6)$. Esta patología puede ser sintomática o asintomática. Los síntomas están presentes desde el nacimiento los cuales pueden ir creciendo hasta producir severas alteraciones vertebrales provocando dolor, adormecimiento, compresión espinal, síndrome de paraplejía y déficit motor (3, 7, 8). Asimismo, no tiene predilección racial, se presenta con ligera predisposición en los varones durante la infancia o la adolescencia, aunque también puede manifestarse en la adultez temprana $(3,7,8)$.

El diagnóstico se establece a partir de la evaluación del tamaño, la apariencia de la piel, los exámenes como la radiografía simple, la resonancia magnética, la tomografía axial computarizada y la angiografía espinal $(1,9)$. Sin embargo, el diagnóstico puede ser difícil debido a que presenta síntomas similares a otras enfermedades como los síndromes de SturgeWeber, de Klippel-Trenaunay-Weber, y de RenduOster-Weber o estar asociada a patologías como la espina bífida oculta y el hemangioma cutáneo (10, 11, 12). El abordaje terapéutico de este síndrome incluye neurocirugía, embolización, radiación, terapia sistémica con corticosteroides (6).
La importancia del diagnóstico temprano de este síndrome se debe a la presencia de las lesiones cutáneo-vasculares que pueden esconder angioma medular acompañado de malformación arteriovenosa (MAV), y generar debilidad o parálisis motora $(1,2)$. Según diversos autores $(1$, 2) en la bibliografía mundial se han publicado menos de cincuenta casos donde la mayor incidencia se da en la infancia y adolescencia; pero la ocurrencia puede ser mayor porque las publicaciones realizadas son de casos sintomáticos. Por ejemplo, Jagla (6) en el 2012 publicó el caso de un recién nacido con asociación coronaria, fístula arterial y catarata congénita. Mientras que, Tubridy (4) en el 2008 reportó un caso con inicio súbito en un infante.

Respecto al abordaje fisioterapéutico, diversos autores han reportado casos de niños que presentaron el síndrome de Cobb y comorbilidades donde el abordaje multidisciplinario (neurología, neurocirugía - embolización) fue similar antes y después de la intervención quirúrgica (2-4). Sin embargo, uno de los casos incluyó la intervención fisioterapéutica que permitió recuperar la fuerza y la movilización, a diferencia de los otros casos (24). De manera que, la intervención multidisciplinaria requiere la inclusión de la fisioterapia para acelerar el proceso de recuperación e incrementar los resultados de forma integral.

Por lo expuesto, el presente reporte de caso clínico tiene como propósito dar a conocer el Síndrome de Cobb en un niño de 5 años de tipo asintomático, cuyo objetivo fue mejorar el proceso de transición de sedente a bípedo. Describiendo las características del síndrome en el niño durante la evaluación y cómo fue la intervención fisioterapéutica, ya que los casos clínicos encontrados en la literatura tienden a describir sólo las características de la enfermedad.

\section{PRESENTACIÓN DEL CASO Datos del paciente}

Paciente de cinco años de edad asistió al servicio de terapia física acompañado de su padre y su abuela materna. El padre informó que el niño nació 
a los nueve meses de gestación, el parto fue por cesárea y se le diagnosticó síndrome de Clippers al nacer. Presentó asimetría en los miembros inferiores (MI), miembro inferior derecho (MID) > miembro inferior izquierdo (MII), con una diferencia de aproximadamente $2 \mathrm{~cm}$. a favor del MID. Actualmente camina y se desplaza distancias largas utilizando ortesis bilateral en el tobillo o la silla de ruedas.

El niño asiste al servicio de terapia física por indicación médica. En abril del 2017 resbaló al bajar las escaleras golpeándose la zona lumbar. Fue intervenido, en un hospital por el hematoma presentado debido a la lesión en los vasos sanguíneos, siendo estos cauterizados por medio de láser. Luego de seis días, fue sometido a una segunda intervención porque presentaba mucho dolor y detectaron el síndrome de Cobb a nivel de la zona lumbar. Fue hospitalizado por treinta días y después de la operación no presentaba control de esfínteres, estuvo con sonda vesical y no podía caminar.

\section{Evaluación y diagnós tico físico funcional}

En el proceso de evaluación y diagnóstico se aplicaron pruebas para la evaluación osteomuscular, neurológica y de piel. Además de la Clasificación Internacional del Funcionamiento de la Discapacidad y de la Salud (CIF).

En la evaluación osteomuscular se encontró:

- Durante la evaluación postural en bipedo se observó que el niño pierde estabilidad con facilidad y para identificar si el problema es tronco o MI se realizaron pruebas de disociación entre las cinturas escapular y pélvica. Se encontró poca estabilidad en el tronco y falta de fuerza en la musculatura de los miembros inferiores (MI).

- Así mismo, en las pruebas de estiramiento pasivo se encontró acortamientos musculares en isquiotibiales, aductores, psoas y cuádriceps. Además en las pruebas de movimiento activo se observó debilidad muscular en el músculo glúteo mayor, glúteo medio y tibial anterior; hiperactividad en el músculo flexor propio del dedo gordo.

- La amplitud articular de los miembros inferiores fue medido con el software Kinovea y es funcional. Se evidenció limitación en la dorsiflexión del tobillo, en MII 7 grados y en MID 8 grados.

- La fuerza muscular en ambos miembros según la escala de Daniel fue: en cadera presenta flexión de grado 3, en abducción de grado 1 y con respecto a extensión, aducción, rotación interna y externa presenta grado 2. En rodilla presentó en flexión y extensión un grado 3. En tobillo plantiflexión y dorsiflexión de grado 2-, inversión y eversión de grado 1 . Asimismo, presentó flexión y extensión de los dedos del pie de grado 2.

- Presenta hipotonía en miembros inferiores (MI) por disminución de fuerza, provocando una alteración motora.

- El trofismo muscular del MII estaba disminuido, evaluado por perimetría (> $2 \mathrm{~cm})$.

- $\mathrm{Al}$ medir la longitud de los MI se encontró una diferencia de $2 \mathrm{~cm}$ debido a que el fémur y la tibia del MII son más cortos que el MID, la medición se realizó con centímetro.

En la evaluación neurológica se encontró lesión a nivel sensitivo en S1, S3, S4, S5 provocando hipoestesia en el dermatoma S1 y anestesia en S3, S4, S5, presentando función vesical e intestinal alterada con pobre control de esfínteres. Asimismo, lesión a nivel motor en S1 por disminución de fuerza muscular.

En la evaluación dérmica se encontró en la zona lumbar una cicatriz por la cirugía practicada, que según la escala de Vancouver presenta pigmentación y coloración normal, flexibilidad con mínima resistencia y altura: $<2 \mathrm{~mm}$. También, se observó en el muslo derecho y partes del glúteo superficies planas pigmentadas de color rojizo, con mayor temperatura que el resto del cuerpo, debido a la MV cutánea. 
Se empleó como herramienta clínica la CIF, que pertenece a la familia de clasificaciones internacionales desarrolladas por la Organización Mundial de la Salud (OMS) diseñada con un propósito múltiple para ser utilizada en diferentes disciplinas y aspectos de la salud. Se analizaron las posturas, la base de soporte y puntos de apoyo, características de movimiento, fuerza, coordinación, limitaciones, sus causas y el análisis de potencialidades para mejorar. También, se consideró los factores contextuales del niño (su participación e interacción con los familiares y en otros ambientes), como se muestra en la tabla 1. equilibrio y coordinación (tabla 2).

\section{2) Intervención}

Se utilizó el método Perfetti porque permite emplear ejercicios cognitivos donde el paciente reconoce el medio, lo interpreta y resuelve a través del movimiento; a su vez desarrolla nuevas habilidades motoras activando los sistemas: sensitivo, perceptivo y motor; asimismo. De igual forma, permite regular el control del tono muscular y recuperar la musculatura que presente déficit a través de las aferencias cinestésicas, barognósicas

Tabla 1. Factores sociales, individuales y funciones motoras

\begin{tabular}{|c|}
\hline Factores sociales \\
\hline $\begin{array}{l}\text { - El niño socializa, expresa sus opiniones abiertamente y se deja entender. } \\
\text { - Su familia es de tipo extendida (constituido por papá, mamá, un hermano de } 13 \text { años y la abuela } \\
\text { materna) } \\
\text { - Asiste a las terapias con el padre y la abuela quien lo cuida. } \\
\text { - En las actividades de la vida diaria (AVD) participa parcialmente en su aseo personal y en el vestido } \\
\text { - En sus alimentos es independiente pero le gusta que le hagan comer. } \\
\text { - Participa en el traslado en sus AVD (cama-silla, silla-silla comedor, silla-inodoro). } \\
\text { - Interactúa con la familia, participa en las actividades del colegio, en las fiestas infantiles y en lugares } \\
\text { - } \text { públicos como supermercados. }\end{array}$ \\
\hline Factores individuales \\
\hline $\begin{array}{l}\text { - Presenta limitación al realizar transición de sentada larga a sentada corta, tiene dificultad porque no } \\
\text { logra hacer maratón y no apoya por completo los pies, busca apoyarse en miembros superiores (MS) y } \\
\text { subir impulsándose con los brazos. } \\
\text { - No logra mantenerse en bípedo, busca apoyarse por lo menos en uno de los miembros superiores, } \\
\text { flexiona rodillas y colapsa. } \\
\text { - Logra mantenerse en bípedo utilizando ortesis de tobillo y pie, pero con muchas compensaciones en } \\
\text { tronco (realiza una hiperextensión de tronco). }\end{array}$ \\
\hline
\end{tabular}

\section{Diagnóstico fisioterapéutico}

Síndrome medular incompleto motor y sensitivo por debajo de S1 y paraplejia flácida.

\section{INTERVENCIÓN}

\section{1) Plan de tratamiento}

El tratamiento fisioterapéutico constó de cinco sesiones con una frecuencia interdiaria y un periodo de $45 \mathrm{~min}$., en las intervenciones se buscó mejorar las condiciones de vida del paciente preparándolo para que pueda desenvolverse de forma independiente en sus AVD, manteniendo su estabilidad y no colapsar debido a la falta de y táctiles que experimenta (13). De igual forma, se empleó el Concepto Bobath para mejorar el control postural, estimulando las reacciones de equilibrio, enderezamiento y defensivas, además de la facilitación de los movimientos con las técnicas de los puntos clave (13).

Se trabajó en forma progresiva empleando el propio peso del paciente y el uso materiales de fisioterapia (pelota bobath, aros, rodillos y banquitos). También se emplearon técnicas de retroalimentación propioceptiva, verbal y de tipo imitación. 


\section{Funciones Motoras}

La postura:

- En sedente se observa la cabeza y hombros en antepulsión, postura cifótica con el tronco en ligera flexión y rotación izquierda, la base de sustentación está en isquion, presentando rotación externa en cadera. Utiliza mayor apoyo en isquion y muslo izquierdo debido a que el pie izquierdo está en plantiflexión con inversión y no lo apoya a diferencia del pie derecho. Se ayuda con los miembros superiores acercándolos al tronco o apoyando la mano o la muñeca. Se encontró poca estabilidad en el tronco y falta de fuerza en la musculatura de los músculos abdominales.

Esta es la postura más alta en el paciente en la cual realizó alcances anteriores, posteriores y laterales. En esta posición logró colocarse el polo y ponerse las medias, pero predominó el apoyo en isquion y muslo izquierdo, mientras que los pies perdieron contacto con el piso sobre todo el lado izquierdo.

- En bípedo con ortesis: cabeza inclinada hacia el lado derecho y rotada hacia el lado izquierdo, con hiperextensión cervical, hombros elevados con predominio al lado izquierdo. Brazos pegados al cuerpo y en ligera extensión. Hiperextensión del tronco acentuándose la curvatura lumbar. Pelvis en anteversión y la cadera derecha en ligera rotación externa. Rodillas en hiperextensión con el pie derecho apoyado en el borde lateral, presentando base de sustentación amplia. Se mantiene en bípedo por corto tiempo con aumento de tono muscular en todo momento.

Transiciones: Transición de sedente a bípedo

- Sin ortésico: lo realizó con dificultad y con ajustes posturales anticipatorios preparatorios y de acompañamiento. Inicia el movimiento apoyando las dos manos sobre la banca, busca apoyo con la mano derecha e inmediatamente realiza ajustes posturales compensatorios para no caer, aumenta el tono muscular, rota el tronco hacia el lado derecho y amplía la base de sustentación.

- Bípedo sin ortésico: se evidenció antepulsión de cabeza, hiperextensión de tronco, pelvis en anteversión, cadera izquierda en flexión y rotación externa con flexión de rodilla y dedos del pie en garra; además, apoya las piernas en los bordes laterales del banco. Se encontró poca estabilidad en el tronco y falta de fuerza en la musculatura de los miembros inferiores (MI) sobretodo en cuádriceps para mantener la extensión de rodilla y por ello se va a una hiperextensión.

- Bípedo con ortésico: lo realizó en el segundo intento, con dificultad, inicia el movimiento apoyando una mano en la rodilla y la otra en la banca. Luego al levantar la pelvis de la banca realiza una aducción de cadera marcada en el lado izquierdo y ambos pies se encuentran en plantiflexión, siendo más marcado en el pie izquierdo. Luego realiza una hiperextensión de rodilla, una anteversión de cadera y una hiperextensión de tronco, pega los brazos al cuerpo y coloca las manos en puño.

Se inició con la recuperación de la elasticidad de los músculos acortados en isquiotibiales, aductores, psoas y cuádriceps. Para ello, se realizaron ejercicios de estiramiento estático

pasivo y pasivo asistido, aplicando una fuerza leve y liberado en forma gradual para evitar el incremento de carga tensora en los tejidos conectivos, mediante tres series y tres repeticiones en cada ejercicio. El propósito fue mejorar la elasticidad $y$ por ende el aumento del rango articular en cadera y rodilla.

Para tratar la hipoestesia moderada en los MI, se empleó la estimulación de la sensibilidad exteroceptiva (piel) realizada con instrumentos de diferentes texturas lisas y rugosas como: algodón, esponjas, cepillos, escobillas, etc. De igual forma, el uso de temperaturas en el recorrido del dermatoma haciendo énfasis en las áreas de apoyo del pie, con la finalidad de estimular el tono de los músculos del pie. Esta información de tacto y temperatura fue percibida por el menor mediante los mecanoreceptores que se encontraron distribuidos en la piel.

La mejora de la sensibilidad propioceptiva se logró con los ejercicios propioceptivos desarrollados en posición sentada corta, de esta manera el niño aumentó el área de contacto del pie realizando presiones y deslizamientos sobre una una pelota y rodillos. Luego en la posición bípeda, se colocó una pelota pilates delante del niño para que flexione el tronco quedando suspendido sobre dicha pelota. La actividad consistió en impulsarse hacia adelante con los pies y realizar el lanzamiento de pelotas pequeñas a un cesto regulando el peso al retornar sobre cada extremidad inferior. 
Para lograr un movimiento corporal adecuado y la carga que debe soportar los pies se empleó el método Perfetti, integrando los ejercicios a los procesos cognitivos. Para esta actividad el paciente estuvo en bipedo con una pelota pilates delante de él; se le indicó que debe flexionar el tronco y recoger un aro para insertarlo en una base colocada primero hacia la derecha y luego hacia la izquierda, en el que debe realizar desplazamientos pequeños y variando la carga en forma alternada en cada MI. equilibrio, enderezamiento y de apoyo, facilitando el movimiento con la técnica de puntos clave (13). Logrando mejorar la posición y la carga de peso en ambos pies, favorecer el traslado de la tibia hacia adelante, activar el cuadricep y los extensores de la cadera. Asimismo, elongar los isquiotibiales, activar los músculos del core, paravertebrales y de la cintura escapular.

Las actividades basadas en mejorar el control postural consistieron en mantener una postura y

Tabla 2. Objetivos, plan de tratamiento y métodos empleados en la intervención

Objetivo general: mejorar el proceso de transición de sedente a bípedo para que el paciente pueda realizar de forma independiente sus AVD.

\begin{tabular}{|c|c|c|}
\hline Objetivos específicos & Plan de tratamiento & Métodos \\
\hline $\begin{array}{l}\text { Recuperar la elasticidad } \\
\text { muscular. }\end{array}$ & $\begin{array}{l}\text { Ejercicios de estiramiento en los músculos acortados } \\
\text { isquiotibiales, aductores, psoas y cuádriceps. }\end{array}$ & $\begin{array}{l}\text { Técnicas de } \\
\text { estiramiento estático } \\
\text { pasivo, pasivo } \\
\text { asistido. }\end{array}$ \\
\hline $\begin{array}{l}\text { Recuperar la } \\
\text { sensibilidad superficial. }\end{array}$ & $\begin{array}{l}\text { Aplicación de ejercicios de estimulación sensorial a través } \\
\text { de la piel, trabajando con superficies de diferentes texturas } \\
\text { y temperaturas sobre los dermatomas afectados. }\end{array}$ & $\begin{array}{l}\text { Estimulación } \\
\text { exteroceptiva. }\end{array}$ \\
\hline $\begin{array}{l}\text { Mejorar la sensibilidad } \\
\text { propioceptiva. }\end{array}$ & $\begin{array}{l}\text { Ejercicios para mejorar la sensibilidad, empleando la } \\
\text { información propioceptiva y exteroceptiva. De esta manera, } \\
\text { se facilita el movimiento y el reconocimiento de las partes } \\
\text { del cuerpo. }\end{array}$ & $\begin{array}{l}\text { Ejercicios | } \\
\text { propioceptivos. } \\
\text { Enfoque de Perfetti. }\end{array}$ \\
\hline $\begin{array}{l}\text { Fortalecer la } \\
\text { musculatura de los MI y } \\
\text { recuperar el trofismo } \\
\text { muscular del MII. }\end{array}$ & $\begin{array}{l}\text { Cargas del propio peso del paciente para aumentar la } \\
\text { tensión muscular sin provocar variantes en la elongación } \\
\text { del músculo, progresando a una resistencia externa manual. } \\
\text { Posteriormente, sostener un peso en una posición } \\
\text { determinada, empujar o tirar de un objeto inamovible. }\end{array}$ & $\begin{array}{l}\text { Ejercicios isométricos } \\
\text { contra resistencia }\end{array}$ \\
\hline
\end{tabular}

Mejor control postural

Ejercicios para mejorar la disociación de la cintura Concepto Bobath para la bipedestación. escapular y de la cintura pélvica. Aplicación de la manipulación para la alineación del calcáneo y regular el tono postural normal. Finalmente, ortesis en el tobillo y pie con un alza para mejorar simetría y estabilidad.

Para fortalecer la musculatura en los MI y recuperar el trofismo muscular del MII se realizaron ejercicios isométricos contra resistencia dándole mayor énfasis a los músculos cuádriceps, glúteo medio, glúteo mayor, aductores, tibial anterior y perineo. Asimismo, se solicitó al paciente que mantenga la extensión de rodilla empleando la carga de su propio peso, luego se agregó una resistencia externa manual suave y finalmente se colocó una pesa de $1 \mathrm{~kg}$ en el tobillo.

Para mejorar el control postural en sedente y en bipedo se emplearon las técnicas del concepto Bobath y se utilizó los ortésicos para mejorar la estabilidad articular en tobillo y pie. Con los ejercicios se buscó controlar las reacciones de desplazamientos del centro de gravedad. Primero, el paciente en posición sentada corta, llevó el tronco hacia adelante, levantó la pelvis, extendió la cadera y las rodillas, flexionó el hombro y mantuvo la posición con los codos extendidos y las manos apoyadas sobre una superficie estable. Luego sobre un rodillo y después sobre una pelota con una duración en cada estructura de diez segundos, al finalizar se retorna a la posición inicial y se repite la secuencia.

En la siguiente actividad el niño mantuvo la postura anterior pero se le pidió que realice deslizamiento de la pelota y el rodillo hacia adelante y hacia atrás, a través de tres series con cinco repeticiones. Por último, se realizó un 
ejercicio manteniendo la posición en bipedo que involucró la activación de los músculos abdominales, la regulación de la posición de la pelvis, el sostenimiento de la extensión de cadera y rodilla y una base de sustentación adecuada al paciente. Los puntos clave de control empleadas en las actividades fueron: cintura escapular, punta del esternón (D7-D8), cintura pélvica y rodillas evitando que el paciente colapse

\section{RESULTADOS}

Después de cinco sesiones de intervención con frecuencia interdiaria por un periodo de 45 minutos se mejoró el proceso de transición de sedente a bipedo, ya que el paciente logró concientizar el apoyo de la superficie de la planta del pie en las posiciones de sedente y bipedo.

Se logró una mayor extensibilidad de la musculatura acortada y por ende mejorar la flexibilidad articular, regular la vasculación de la pelvis que se encontraba en anteversión debido al acortamiento del músculo cuadriceps y la hiperextensión de la columna por compensación.

Respecto a la sensibilidad superficial se aumentó la percepción sobre los estímulos (texturas) en los bordes laterales del pie. Asimismo, se mejoró la sensibilidad propioceptiva en general, sobre todo en tobillo, rodilla y cadera debido a que mejoró el reconocimiento de la posición de estas para controlar la postura y de esta manera se ganó estabilidad.

Se ganó mayor fuerza muscular en cuadriceps (4/5) para una extensión de rodilla y en glúteos (3/5) para lograr la transición de sedente a bipedo y mantener esta última postura. A su vez mejoró el trofismo de los músculos afectados.

En cuanto al control postural en posición sentada corta, se mejoró el apoyo bilateral en isquion, muslo, y los pies ya no pierden totalmente contacto con el piso. Asimismo, durante la progresión de sedente a bipedo reguló el aumento de tono muscular; así como, las reacciones de equilibrio, de enderezamiento y de apoyo. De esta manera, logró una mayor estabilidad en la posición bipedo. Además; los ortésicos ayudaron a mejorar la estabilidad articular en tobillo y pie.

\section{DISCUSIÓN}

El presente reporte de caso tuvo como objetivo mejorar la transición de sedente a bipedo y la bipedestación del paciente para lograr una mayor independencia en sus AVD. Asimismo, registrar las características que presentó un niño con Síndrome de Cobb, ya que existen pocos casos expuestos debido a su condición asintomática, comprometiendo al sistema nervioso y provocando una diplejia.

Se logró concientizar el apoyo de la planta del pie y por ende mejorar la base de sustentación, el equilibrio, la estabilidad y la postura en las posiciones de sedente y bipedo. En la recuperación del control motor se integraron los tres niveles de jerarquía: la médula, el tronco encefálico y la corteza cerebral; encargados de regular la locomoción, el control y el mantenimiento de la postura. También, se buscó el movimiento normal integrando la información sensorial que llega al SNC y la respuesta que se ejecuta en forma de orden motora. En este caso el movimiento corporal se vio alterado por la instauración de un tono postural anormal y patológico; además de la alteración de la sensibilidad y los receptores somatosensitivos, por lo que no permitieron que haya un movimiento normal (13).

Se recuperó la elasticidad de los músculos acortados permitiendo mejorar la flexibilidad articular y la postura. Al respecto, se conoce que los estiramientos son maniobras terapéuticas que tiene por finalidad elongar estructuras de tejido blando acortadas y los beneficios que ofrece son: reducir la tensión muscular, relajar el cuerpo, mejorar la coordinación, facilitar y aumentar la movilidad para prevenir lesiones (14).

Asimismo, los ejercicios de estiramiento de baja intensidad asociadas a una larga duración producen una mejoría de la amplitud de movimiento sin exponer a los tejidos (14). El entrenamiento de la flexibilidad con el método pasivo estático es una elección eficaz y segura (15). Además, la aplicación sistemática de programas de estiramiento consiguen mejoras en la flexibilidad (16).

Respecto a la hipoestesia moderada tratada mediante la estimulación de la sensibilidad exteroceptiva, se logró el aumento del apoyo en el pie izquierdo. Este resultado fue posible a través de la aplicación de estímulos externos percibidos por los mecanoreceptores que obedecen a las 
terminaciones nerviosas libres y a las terminaciones encapsuladas (corpúsculos de Meissner, Ruffini y Pacini) (2, 17). Debido a que, las primeras permiten detectar el dolor y por otro lado, las terminaciones encapsuladas perciben estímulos diferentes. Los corpúsculos de Meissner localizan la sensibilidad para el tacto fino, los discos de Merckel detectan el tacto superficial, los de Pacini ubican las vibraciones rápidas y la presión mecánica profunda, y las de Ruffini perciben los cambios de temperatura relacionados con el calor $(2,17)$.

En cuanto a la sensibilidad propioceptiva, el niño aumentó su percepción sobre los estímulos en los bordes laterales del pie y el reconocimiento de la posición correcta para el control postural. Este resultado se explicaría en el empleo del método Perfetti para reeducar la sensibilidad propioceptiva que consiste en ejercicios terapéuticos cognitivos mediante la activación de los sistemas sensitivo, perceptivo y motor (13). Asimismo, las aferencias cinestésicas, barognósicas y táctiles permitieron conseguir el control del tono muscular y la recuperación de la musculatura deficitaria (13).

Respecto a la hipotonía y la disminución del trofismo muscular del MII, se realizaron ejercicios isométricos contra resistencia para fortalecer la musculatura de los MI y la recuperación del trofismo del MII. Los ejercicios isométricos permiten realizar en forma estática la contracción del músculo sin cambios perceptibles en la longitud de este y sin movimientos articulares visibles, produciendo una magnitud mensurable de tensión y fuerza (14). De manera que, es un método efectivo para mejorar la fuerza muscular (14).

Por último, se mejoró el control postural para la posición en sedente y bipedo a través de la aplicación del Concepto Bobath. Este resultado coincide con lo reportado en la literatura sobre la aplicación de la terapia Bobath que se basa en el control del tono postural, la inhibición de patrones de actividad refleja, la facilitación de patrones motores normales y el control funcional efectivo (13). Sumado a ello, se deben considerar las actividades realizadas en la intervención que incluyeron reacciones de enderezamiento, de equilibrio y defensivas. Asimismo, debido a la elección de una base de sustentación adecuada al tono postural del paciente, ya que desde el punto de vista físico, el tono muscular es inversamente proporcional a la base de sustentación y al apoyo (13).

La duración del tratamiento de cinco sesiones fue una limitación debido al proceso lento de recuperación en el niño. Las complicaciones que presentó a partir de la última intervención quirúrgica interfirieron en las terapias, ya que tuvo que adecuarse las actividades y ejercicios al ritmo de su recuperación. Frente a ello, fue importante el compromiso de la familia de asistir a las terapias de forma regular y complementar con los ejercicios en casa.

Por otro lado, se destacan los resultados obtenidos en solo cinco sesiones. Esto fue posible gracias al diseño de intervención fisioterapéutica y el adecuado diagnóstico. Por lo cual, es necesario que el profesional incluya el diagnóstico diferencial como parte de los criterios para arribar a un diagnóstico. Puesto que, el síndrome de Cobb comparte ciertas características con el síndrome de Clippers y su oportuna identificación hubiese permitido iniciar el tratamiento fisioterapéutico en una edad más temprana y probablemente la prevención de comorbilidad.

Se recomienda en futuras investigaciones profundizar el abordaje fisioterapéutico con otras técnicas que sumen al tratamiento. Además de planificar estrategias de prevención y de rehabilitación para evitar malformaciones óseas que puedan complicar el abordaje y la recuperación del paciente en la ejecución de sus actividades de la vida diaria. Finalmente, se sugiere a los fisioterapeutas que reporten no sólo las características del síndrome sino también el abordaje fisioterapéutico.

\section{CONCLUSIONES}

La intervención fisioterapéutica permitió mejorar el proceso de transición de sedente a bípedo, logrando recuperar la funcionalidad de la musculatura de los MI, la sensibilidad propioceptiva y el control postural. Por otra parte, la aplicación de la técnica Perfetti y el Concepto Bobath permitieron la activación de los músculos abdominales y la regulación de las reacciones de equilibrio, enderezamiento y de apoyo. Todo ello 
permitió que el niño sea más independiente respecto a la movilización y el desarrollo de las actividades de la vida diaria. Lo cual demuestra que la eficacia de la terapia a nivel físico puede desencadenar una mejora en la calidad y estilo de vida del paciente.

\section{REFERENCIAS BIBLIOGRÁFICAS}

1. Álvarez J, Álvarez E, Clint JL, Sauret J .Angiomatosis 7. Moscote LR, Escorcia H, Feris R. cutaneovertebral medular (síndrome de Cobb). Revista Neurol. 2008;47(11):613-614.

2. Palanca D, Rius N, García G, 8 . Cadela S, Sola Martínez T. y Palomeque A, Síndrome de cobb o angiomatosis cutáneo-meningoespinal. In Anales de Pediatría. 2010;73( 2):109-111.

3. Pal P, Ray S, Chakraborty S, Dey S, Talukdar A. Cobb syndrome: A rare cause of paraplegia. Annals of 9 . Neurosciences. 2015;22(3):191193.

4. Clark MT, Brooks EL, Chong W, Pappas C, Fahey M. Cobb syndrome: a case report and systematic review of the literature. Pediatric 2008;39(6):423-425.

5. Brant AJ, James HE, Tung $\mathrm{H}$ Cutaneomeningospinal

Angiomatosis (Cobb Syndrome) Wuth Tethered Cord. Pediatric Neurosurgery. 1999;30:93-95.

6. Jagła M, Szymońska I, Kruczek P. Sonographic findings in a neonate with Cobb syndrome. Journal of Clinical Ultrasound. 2013;41(4):258-260
12. Canneti B, Mosqueira AJ, Gilo F, Carreras T, Barbosa A, MecaLallana V, Vivancos J. Síndrome CLIPPERS con distribución atípica de las lesiones en la resonancia magnética cerebral.

Krolak-Salmon P, Moreau T, Rev Neurol. 2013;57(8): 354-358.

Bouhour F, Bascoulergues Y

Secchi T, Confavreux C, 13. Bisbe M, Santoyo C; Segarra VT. Simultaneous Medullar and Fisioterapia en Neurología. Cutaneous Revelation of a Panamericana. Argentina. 2012.

Cutaneomeningospinal

Angiomatosis. Eur Neurol. 14. Kisner C y Allen CL. Estiramiento 1999;41(3):170-171.

Cejas C, Escobar I, Serra M. y Barroso F. Neurografía de alta resolución del plexo lumbosacro en resonancia magnética $3 \mathrm{~T}$. Revista Elsevier Doyma. 2015;57(1):22-34.

10. Gao $\mathrm{P}$, Zhang $\mathrm{H}$, Ling $\mathrm{F}$. Angiogenic and inflammatory factor expressions in cutaneomeningospinal angiomatosis (Cobb's syndrome): Case Report. Acta Neurochir: 16. Ayala F, Sainz PA, Cejudoc A. El 2011; 153(8):1657-1661.

11. Romero-Cabello R, RomeroFeregrino R, Romero-Feregrino R, Sánchez CJ, Lazos-Ochoa M. Angiomatosis bacilar: reporte de 17. García JA, Hurlé JM. un caso. Rev Latinoam Patol Clin Med Lab. 2016;63(3):119-123. para los trastornos de la movilidad. Ejercicios Terapéuticos: Fundamentos y técnicas. Buenos Aires. En: Editorial Médica Panamericana. 5ta ed. 2010: 65108

15. Chulvi I.; Masiá L. La flexibilidad criterios básicos para su entrenamiento saludable aplicando la metodología pasivo estática: Journal of Sport and Health Research. 2012;4(1):11-22. entrenamiento de la flexibilidad: técnicas de estiramiento: Rev Andal Med Deporte. 2012;5(3):105-112.

Neuroanatomía Humana. Ed. Panamericana 2015 